\title{
Competição em Preços entre Corretores de Seguros de Automóveis
}

\section{Bruno Cesar Aurichio Ledo}

Submetido: 24 de junho de 2010; aceito para publicação: 05 de outubro de 2011

Resumo: O preço final em um contrato de seguro de automóvel não depende apenas da seguradora, mas também do corretor de seguros. Sendo assim, o corretor é capaz de alterar o mecanismo desenhado inicialmente pela seguradora, distorcendo a alocação do risco entre as partes. Este artigo adapta o modelo de Stahl (1989) ao mercado de seguros de automóveis com o objetivo de explicar o comportamento estratégico dos corretores. O modelo prevê que o valor esperado da taxa de comissão escolhida pelo corretor seja função decrescente do prêmio requerido pela seguradora. Esta previsão foi testada e confirmada empiricamente, utilizando dados sobre vendas de apólices no mercado brasileiro. Na prática, este artigo contribui para que as seguradoras tenham maior controle sobre o prêmio final do seguro, o que torna a alocação do risco mais eficiente neste mercado.

\begin{abstract}
The final price in a contract of automobile insurance depends not only the insurance company, but also the insurance agent. Thus, the agent is able to change the mechanism initially designed by the insurer, distorting the allocation of risk between the parts. This article adapts the model of Stahl (1989) to automobile insurance market in order to explain the agent's strategic behavior. The model predicts that the expected value of the commission rate chosen by the agent is a decreasing function of the premium required by the insurer. This prediction was tested and confirmed empirically using data on sales of policies in the Brazilian market. In practice, this article gives to the insurers more control over the final price, which drives to a more efficient allocation of risk in this market.
\end{abstract}

Bruno Cesar Aurichio Ledo

Doutor em Economia FEA-RP/USP - Endereço para contato: Av. Bandeirantes, 3900 Monte Alegre - CEP 14040-905 - Ribeirão Preto/SP - E-mail: brunoaurichio@gmail.com 


\section{Introdução}

O mercado de seguros de automóveis é composto por três agentes econômicos: a seguradora, o segurado e o corretor de seguros. O corretor de seguros é o intermediário legal entre a seguradora e o segurado. Cabe ao corretor apresentar ao segurado os diferentes contratos disponibilizados pelas seguradoras e garantir que seus termos sejam cumpridos por ambas as partes.

Embora o corretor seja determinante na relação entre seguradora e segurado, seu papel tem sido negligenciado pela teoria econômica. Tomemos como exemplo os modelos de seleção adversa, nos quais as seguradoras escolhem cuidadosamente combinações de prêmios e coberturas de modo a incentivar os segurados a autosselecionarem os contratos em função de seu risco ex ante. Tais modelos pressupõem que as seguradoras sejam capazes de fixarem, sozinhas, o prêmio final do seguro. No entanto, o prêmio final pode variar bastante em função da taxa de comissão escolhida pelo corretor no ato da venda. ${ }^{1}$ Sendo assim, o corretor é capaz de distorcer os incentivos implícitos contidos no contrato desenhado inicialmente pela seguradora, gerando ineficiência na alocação do risco entre as partes.

Nos dados brasileiros sobre o mercado de seguros de automóveis se observa um fato estilizado: uma elevada dispersão nas taxas de comissão escolhidas pelos corretores, mesmo quando diversos fatores observáveis são controlados. O objetivo deste artigo é compreender o processo gerador dessas taxas de comissão e seus principais determinantes. Para tal, apresenta-se uma versão do modelo de Stahl (1989) adaptada ao mercado de seguros de automóveis. Nele, segurados heterogêneos buscam sequencialmente pela menor taxa de comissão entre corretores homogêneos. A cada nova busca os segurados incorrem em um custo fixo de procura. O custo de procura é uma informação privada dos segurados. A presença de segurados com custo zero implica que o equilíbrio de Nash ocorra em estratégias mistas, gerando dispersão nas taxas de comissão tal como observado empiricamente.

\footnotetext{
${ }^{1} \mathrm{O}$ prêmio final a ser pago pelo segurado pode ser decomposto em duas partes: a parte da seguradora e a parte do corretor. A parte da seguradora é fixa. Já a parte do corretor depende da taxa de comissão que ele próprio escolher no ato da venda do contrato. Sendo assim, quanto maior a taxa de comissão escolhida pelo corretor, maior o prêmio final pago pelo segurado.
} 
No modelo, a função distribuição das taxas de comissão depende dos seguintes parâmetros: valor do custo de procura, proporção de segurados com custo zero de procura, número de corretores no mercado e prêmio mínimo requerido pela seguradora. Os três primeiros parâmetros já foram bastante explorados no campo da organização industrial. No entanto, pouco se sabe sobre a influência que o prêmio da seguradora exerce sobre as estratégias dos corretores e, consequentemente, sobre o prêmio final do seguro.

O modelo prevê que o valor esperado da taxa de comissão seja função decrescente do prêmio mínimo requerido pela seguradora. A intuição é que quanto maior o prêmio fixado pela seguradora, maior o benefício esperado do segurado em procurar uma nova oferta. Em função disso, os corretores passam a competir de forma mais agressiva, diminuindo o valor esperado de sua taxa de comissão.

Para testar a previsão do modelo regrediu-se a taxa de comissão (em nível e logaritmo) contra o logaritmo do prêmio mínimo requerido pela seguradora, além de um conjunto de variáveis observáveis pelo corretor no instante em que escolhe sua taxa de comissão (características do segurado, do automóvel, do contrato e do mercado).

A base de dados utilizada neste artigo foi elaborada pela SUSEP (Superintendência de Seguros Privados), autarquia responsável por regular o mercado de seguros no Brasil. Os dados referem-se aos contratos de seguros de automóveis vendidos no Brasil entre os anos de 2002 e 2004. Trata-se de um agrupamento de cortes transversais, uma vez que é impossível construir um painel com os dados da SUSEP (não se tem acesso ao nome do segurado, ou qualquer outra chave que permita acompanhar o mesmo contrato ao longo do tempo).

O mercado brasileiro de seguros de automóveis é dividido em 41 classes de localização de acordo com critérios geográficos definidos pela SUSEP. A mesma metodologia de teste foi implementada separadamente nas 41 classes de localização. Os resultados se mostraram bastante robustos e corroboraram de modo significativo a previsão do modelo. Nas regressões da taxa de comissão em logaritmo a previsão do modelo se verificou em todas as regiões. Já nas regressões da taxa de comissão em nível, a previsão do modelo se confirmou em 39 das 41 regiões. 
$\mathrm{Na}$ classe de localização referente à Região Metropolitana de São Paulo, por exemplo, os testes indicaram que para cada $1 \%$ de aumento no prêmio mínimo da seguradora, a taxa de comissão do corretor diminui 0,023 pontos percentuais, em média. A implicação prática deste resultado é que caso a seguradora deseje aumentar o prêmio final em 1\% (para melhor alocar o risco, por exemplo) terá que aumentar o prêmio requerido em mais de $1 \%$. Na prática, este resultado contribui para que as seguradoras minimizem as distorções que o corretor pode causar no contrato (via preço).

O restante deste artigo está dividido da seguinte maneira: a Seção 2 apresenta um resumo da literatura sobre dispersão de preços. A Seção 3 oferece um modelo para explicar como corretores competem em taxas de comissão. A Seção 4 apresenta a análise empírica do artigo, onde se encontra a descrição da base de dados, algumas estatísticas descritivas da amostra, a metodologia de teste e os principais resultados obtidos. A Seção 5 apresenta a conclusão do artigo.

\section{Literatura sobre Dispersão de Preços}

Dispersão de preços para bens homogêneos pode ser causada por diversos fatores, tais como custos heterogêneos das firmas, informação imperfeita e custos de procura (search costs). Esta seção apresenta uma breve revisão da literatura sobre dispersão de preços.

Em artigo pioneiro, Stigler (1961) explica a existência de dispersão de preços propondo um modelo de search com informação imperfeita. Stigler (1961) argumenta que a dispersão de preços depende da intensidade de search por parte dos consumidores e que esta intensidade será maior quando o gasto com o bem representar uma elevada fração da renda, as compras forem frequentes e o custo de search for baixo. A principal crítica feita a este trabalho é que a dispersão de preços é exógena ao comportamento das firmas, pois ignora a possibilidade das firmas anteciparem o comportamento dos consumidores e escolherem seus preços otimamente.

Ao levar em consideração o comportamento otimizador das firmas em um mercado com informação imperfeita e custos positivos de search, Diamond (1971) mostrou que o equilíbrio de Nash será único 
e tal que todas as firmas escolherão o preço de monopólio. Este resultado, conhecido como o "Paradoxo de Diamond", lançou o desafio teórico de explicar a descontinuidade entre o preço competitivo e o preço de monopólio em modelos de search.

No modelo proposto por Reinganum (1979), firmas com custos heterogêneos escolhem preços que são meros markups sobre seus custos, resultando assim em dispersão de preços. Outros artigos também assumem custos heterogêneos entre as firmas (ver MacMinn (1980), Carlson e McAfee (1983) e Benabou e Gertner (1993)) e derivam seus resultados de dispersão de preços assumindo que os consumidores possuem custo positivo de search e, além disso, não observam os custos das firmas.

No entanto, custo heterogêneo não é condição necessária para gerar dispersão de preços. Burdett e Judd (1983) foram pioneiros ao mostrar que dispersão de preços pode ocorrer mesmo na presença de firmas e consumidores homogêneos.

Stahl (1989) propõe um modelo com firmas homogêneas e consumidores heterogêneos. Em seu modelo de search sequencial há dois tipos diferentes de consumidores: os chamados "shoppers", com custo zero de procura, e os demais com custo positivo. As firmas não observam o tipo do consumidor. O equilíbrio é único e tal que a distribuição de preços depende da proporção de "shoppers" no mercado. Se todos os consumidores forem "shoppers", então a distribuição será degenerada no preço competitivo. Por outro lado, se todos os consumidores possuírem custo positivo de procura, então a distribuição será degenerada no preço de monopólio. A distribuição muda suavemente de um extremo a outro em função da proporção de "shoppers", explicando assim o "Paradoxo de Diamond".

A literatura empírica recente tem estimado algumas estáticas comparativas previstas pelos modelos teóricos como forma de testar se a dispersão de preços observada é, de fato, consequência da existência de search nos mercados. Sorensen (2000), por exemplo, estuda a dispersão de preços no mercado de remédios e obtém que a intensidade de search é maior em caso de medicamentos de uso continuado. Neste caso, o maior empenho de procura no presente será compensado por um preço mais baixo no decorrer do tempo. 
Outra questão empírica relevante diz respeito ao número de vendedores em um mercado ("seller density"). Utilizando dados sobre o mercado de revenda de gasolina em quatro regiões diferentes dos Estados Unidos, Barron et al. (2004) estimaram que o nível de preços e sua dispersão decrescem em função do número de vendedores no mercado. Este resultado, no entanto, é contestado por Chandra e Tappata (2009), que obtiveram que a dispersão de preços aumenta em função do número de revendedores de gasolina.

Dahlby e West (1986) estudaram a dispersão de preços existente no mercado de seguros de automóveis. Em particular os autores encontraram que a variância nos prêmios de seguro decresce com o número de companhias seguradoras no mercado e concluíram que este resultado depende fundamentalmente da existência de custos de search entre os segurados.

A literatura empírica sobre dispersão de preços entre corretores de seguros é bastante escassa. Pretende-se com o presente artigo tentar reduzir esta lacuna.

\section{Modelo}

Esta seção apresenta uma versão do modelo de Stahl (1989) adaptada ao mercado de seguros de automóveis. Em particular, condicionamos as estratégias dos corretores ao prêmio requerido pela seguradora e derivamos um resultado testável sobre o valor esperado das taxas de comissão dos corretores.

Considere uma economia povoada por seguradoras, corretores e segurados, em que todos vivem um único período. As seguradoras desenham contratos de seguro escolhendo uma cobertura e um prêmio. Como nosso objetivo é estudar a relação entre o corretor e o segurado, assumiremos que haja um único contrato no mercado, com cobertura fixada e prêmio requerido pela seguradora igual a $\mathrm{P}>0$.

Nesta economia há $\mathrm{N}$ corretores idênticos. Por simplicidade, assumimos que o papel do corretor seja simplesmente vender contratos. Como forma de remuneração pela venda, cada corretor escolhe uma taxa de comissão $\alpha \in \mathrm{P}$ sobre o prêmio P. Assim, a receita do corretor será $\alpha \mathrm{P}$ caso o contrato seja vendido e o prêmio total pago pelo se- 
gurado será $(1+\alpha) P$. Assumimos que não há restrição de oferta e que o custo marginal por atender um segurado seja zero.

Os segurados desejam comprar contratos e, para tal, buscam sequencialmente pela melhor oferta entre os corretores. Os segurados são heterogêneos. $\mathrm{O}$ vetor $\mathrm{x} \in \mathrm{P}^{J}$ descreve as características observáveis de cada segurado. Procurar uma nova oferta entre os corretores é uma atividade custosa para o segurado. Uma fração $\mu_{\mathrm{x}} \in(0,1)$ dos segurados possui custo zero de procura, enquanto os $\left(1-\mu_{\mathrm{x}}\right)$ restantes incorrem em um custo fixo $c_{x}>0$. Assumimos que a primeira visita seja grátis e que, a qualquer momento, o segurado possa voltar atrás e resgatar uma proposta passada.

Como veremos adiante, a presença de segurados com custo zero de procura implica que o equilíbrio de Nash, caso exista, dar-se-á em estratégias mistas. Assim sendo, seja $\Delta$ o espaço de funções distribuição em $\mathrm{P}$, nosso objetivo é encontrar uma distribuição $\mathrm{F}_{\mathrm{x}}(\alpha) \in \Delta$ que caracteriza um equilíbrio de Nash.

Começaremos analisando o problema dos segurados. Ao visitar um corretor e receber uma proposta de prêmio $(1+\alpha) \mathrm{P}$, o segurado pode aceitá-la ou procurar uma nova oferta - incorrendo em seu custo de procura. Assim sendo, o benefício líquido esperado pelo segurado em procurar um novo corretor será:

$$
H_{x}(\alpha) \equiv P \int_{b_{x}}^{\alpha}(\alpha-\tilde{\alpha}) d F_{x}(\tilde{\alpha})-c_{x}
$$

sendo $b_{x}$ o limite inferior do suporte de $F_{x}(\alpha)$. Integrando (1) por partes, obtemos:

$$
H_{x}(\alpha) \equiv P \int_{b_{x}}^{\alpha} F_{x}(\tilde{\alpha}) d \tilde{\alpha}-c_{x}
$$

Assumimos que o segurado seja neutro ao risco na busca por novas ofertas. Então, procurar uma nova oferta será vantajoso caso $H_{x}(\alpha)>0$.

Definição 1: Chamaremos de taxa de comissão reserva, $r_{x}$, a taxa pertencente ao suporte de $\mathrm{F}_{\mathrm{x}}(\alpha)$ que faz $\mathrm{H}_{\mathrm{x}}\left(\mathrm{r}_{\mathrm{x}}\right)=0$. 
Uma vez que $H_{x}(\alpha)$ é estritamente crescente em $\alpha$, caso exista, $r_{x}$ será única. Declaramos, então, a regra de decisão ótima entre os segurados: Aqueles com custo $c_{\mathrm{x}}>0$ devem procurar um novo corretor enquanto a menor taxa de comissão observada for maior que $r_{x}$. Segurados com custo $c_{x}=0$ devem visitar todos os corretores e escolher a menor taxa de comissão observada.

Agora, passaremos a estudar o problema dos corretores. Procederemos da seguinte maneira: (i) dada uma taxa de comissão reserva exógena $r_{x}$, encontraremos um equilíbrio de Nash condicional $\mathrm{F}_{\mathrm{x}}\left(\alpha \mid \mathrm{r}_{\mathrm{x}}\right)$; (ii) esta distribuição produzirá alguma comissão reserva $\mathrm{r}_{\mathrm{x}}{ }^{*}$, e; (iii) o equilíbrio estará definido se $r_{x}=r_{x}{ }^{*}$.

Lema 1: Seja $F_{x}\left(\alpha \mid r_{x}\right)$ um equilíbrio de Nash condicional e $B\left(r_{x}\right)$ o limite superior de seu suporte, então $B\left(r_{x}\right)=r_{x}$.

Demonstração. Suponha $\mathrm{B}\left(\mathrm{r}_{\mathrm{x}}\right)<\mathrm{r}_{\mathrm{x}}$, neste caso cobrar $\alpha=\mathrm{B}\left(\mathrm{r}_{\mathrm{x}}\right)+\varepsilon$ domina estritamente $B\left(r_{x}\right)$, uma vez que o corretor não perde qualquer venda e ainda aumenta seu lucro esperado. Suponha $B\left(r_{x}\right)>r_{x}$, neste caso cobrar $\alpha=r_{x}$ domina estritamente $B\left(r_{x}\right)$, uma vez que o corretor sempre realizará a venda para os segurados com custo positivo.

O Lema 1 implica que, em equilíbrio, todos os segurados com custo de procura $c_{x}>0$ aceitarão a primeira taxa de comissão que observarem. Apenas os segurados com custo $c_{x}=0$ realmente farão pesquisa de preços.

Ao receber a visita de um segurado e sortear uma taxa de comissão $\alpha$, o lucro esperado do corretor será

$$
E_{x}[\pi(\alpha)] \equiv\left\{\mu_{x}\left[1-F_{x}\left(\alpha \mid r_{x}\right)\right]^{N-1}+\left(1-\mu_{x}\right)\right\} \alpha P
$$

Definição 2: Uma distribuição $F_{x}\left(\alpha \mid r_{x}\right) \in \Delta$ será um equilíbrio de Nash condicional se

$$
E_{F_{x}}[\pi(\alpha)] \geq E_{F_{x}^{\prime}}[\pi(\alpha)], \forall F_{x}^{\prime} \in \Delta
$$

Lema 2: Uma distribuição $\mathrm{F}_{\mathrm{x}}\left(\alpha \mid \mathrm{r}_{\mathrm{x}}\right) \in \Delta$ será um equilíbrio de Nash condicional se existir $\pi_{\mathrm{x}}$ tal que 


$$
\begin{aligned}
& E_{F_{x}}[\pi(\alpha)]=\bar{\pi}_{x}, \forall \alpha \in \operatorname{supp} F_{x} \\
& E_{F_{x}}[\pi(\alpha)] \leq \bar{\pi}_{x}, \forall \alpha \notin \in \operatorname{supp} F_{x}
\end{aligned}
$$

Demonstração. Suponha que as condições (5) e (6) sejam válidas, mas que $F_{x}$ não seja um equilíbrio de Nash como definido em (4). Então deve existir $F^{\prime} \in \Delta$, tal que $\mathrm{E}_{\mathrm{F}^{\prime}}[\pi(\alpha)]>\mathrm{E}_{\mathrm{Fx}}[\pi(\alpha)]$. Sendo assim, existem estratégias em $\mathrm{R} \backslash \operatorname{suppF}_{\mathrm{x}}$ com massa positiva sob $\mathrm{F}^{\prime}$ que geram payoff esperado maior do que $\pi_{\mathrm{x}}$, o que contradiz (6).

Seja $\pi_{\mathrm{x}}=\left(1-\mu_{\mathrm{x}}\right) \mathrm{r}_{\mathrm{x}} \mathrm{P}$. Então, usando o Lema 2 obtemos

$$
F_{x}\left(\alpha \mid r_{x}\right)=1-\left[\left(\frac{1-\mu_{x}}{\mu_{x}}\right)\left(\frac{r_{x}}{\alpha}-1\right)\right]^{\frac{1}{N-1}}
$$

para todo $\alpha \leq r_{x}$. É fácil ver que $F_{x}\left(\alpha \mid r_{x}\right)$ é estritamente crescente em $\alpha$ e $F_{x}\left(r_{x} \mid r_{x}\right)=1$. Seja $b\left(r_{x}\right)$ o limite inferior do suporte de $F_{x}\left(\alpha \mid r_{x}\right)$. Então, $\mathrm{F}_{\mathrm{x}}\left(\mathrm{b}\left(\mathrm{r}_{\mathrm{x}}\right) \mid \mathrm{r}_{\mathrm{x}}\right)=0$ implica:

$$
b\left(r_{x}\right)=\left(1-\mu_{x}\right) r_{x}
$$

Derivando a Equação (7) com respeito à $\alpha$ obtemos sua função densidade dada por

$f_{x}\left(\alpha \mid r_{x}\right)=\left(\frac{r_{x}\left(1-\mu_{x}\right)}{\alpha^{2} \mu_{x}(N-1)}\right)\left[\left(\frac{1-\mu_{x}}{\mu_{x}}\right)\left(\frac{r_{x}}{\alpha}\right)\right]^{-\left(\frac{N-2}{N-1}\right)}$

que é não negativa em seu suporte.

Para que a caracterização do equilíbrio esteja completa, resta apenas encontrar $r_{x}{ }^{*}$ que satisfaça

$$
H_{x}\left(r_{x}^{*}\right) \equiv P \int_{b\left(r_{x}^{*}\right)}^{r_{x}^{*}} F_{x}\left(\alpha \mid r_{x}^{*}\right) d \alpha-c_{x}=0
$$


Lema 3: $\partial \mathrm{H}_{\mathrm{x}} / \partial \mathrm{r}_{\mathrm{x}}>0$ para todo $\mathrm{r}_{\mathrm{x}}$.

Demonstração.

$\frac{\partial H_{x}\left(r_{x}\right)}{\partial r_{x}}=P\left[1+\int_{b\left(r_{x}\right)}^{r_{x}} \frac{\partial F_{x}\left(\alpha \mid r_{x}\right)}{\partial r_{x}} d \alpha\right]=P\left[1+\int_{b\left(r_{x}\right)}^{r_{x}} \frac{\alpha}{r_{x}} f_{x}\left(\alpha \mid r_{x}\right) d \alpha\right]>0$

O Lema 3 garante que caso exista uma raiz que solucione (10), esta será única. Então, definimos a taxa de comissão reserva coerente com o equilíbrio de Nash como

$$
r_{x} \equiv \begin{cases}r_{x}^{*}, & \text { se } H_{x}\left(r_{x}^{*}\right)=0 \\ +\infty, & \text { caso contrário }\end{cases}
$$

A taxa de comissão reserva definida em (11) gera uma única função distribuição definida em (7) que, por sua vez, reproduz a mesma taxa de comissão reserva - temos então um equilíbrio de Nash. É importante salientar que o equilíbrio será função dos parâmetros do modelo: $\mu_{\mathrm{x}}, \mathrm{C}_{\mathrm{x}}, \mathrm{N}$ e P.

Uma vez determinado o equilíbrio do modelo, nosso objetivo agora é verificar como o prêmio $\mathrm{P}$ fixado pela seguradora afeta a decisão estratégica do corretor através da função distribuição de equilíbrio $\mathrm{F}\left(\alpha \mid \mu_{\mathrm{x}}, \mathrm{C}_{\mathrm{x}}, \mathrm{N}, \mathrm{P}\right)$.

Lema 4: Em equilíbrio, o valor esperado das taxas de comissão decresce com o prêmio fixado pela seguradora.

Demonstração. A condição (10) e o Lema 3 implicam $\partial \mathrm{r}_{\mathrm{x}} / \partial \mathrm{P}<0$. Isto significa que os limites superior e inferior do suporte de $\mathrm{F}$ decrescem com P. Adicionalmente, de (7) temos que:

$$
\frac{\partial F_{x}}{\partial P}=\frac{\partial F_{x}}{\partial r_{x}} \frac{\partial r_{x}}{\partial P}>0
$$

Este resultado implica que a função distribuição $\mathrm{F}$ se desloca para cima quando $\mathrm{P}$ aumenta, ou seja, os corretores sorteiam taxas de comissão menores com maior probabilidade. Portanto, quando o prêmio $\mathrm{P}$ aumenta, a função distribuição das taxas de comissão se desloca para cima e os limites de seu suporte diminuem. Estes dois resultados combinados implicam que o valor esperado da taxa de comissão decresce 
em função do prêmio. A intuição do Lema 4 é a seguinte: quanto maior o prêmio fixado pela seguradora, maior o benefício líquido do segurado em procurar uma nova oferta. Em função disso, os corretores passam a competir de forma mais agressiva, sorteando taxas de comissão menores. Testaremos este resultado na Seção 5.

\section{Análise Empírica}

\subsection{Base de Dados}

A base de dados utilizada neste trabalho foi elaborada pela SUSEP (Superintendência de Seguros Privados), autarquia vinculada ao Ministério da Fazenda e responsável pela regulação do mercado de seguros no Brasil. Semestralmente a SUSEP colhe dados sobre a população dos contratos de seguros de automóveis vigentes no mercado brasileiro. Para cada contrato, há informações sobre coberturas, prêmios, sinistros, bem como características do segurado e do veículo.

A amostra analisada neste trabalho é composta por contratos de seguros de automóveis vendidos no mercado brasileiro entre janeiro de 2002 e julho de 2004. Com os dados fornecidos pela SUSEP não foi possível a construção de um painel, isto porque não há qualquer chave que possibilite identificar um mesmo segurado (ou contrato) ao longo do tempo. Sendo assim, os dados constituem um agrupamento de cortes transversais para 5 semestres.

A amostra considera apenas apólices de pessoas físicas com um único item assegurado (com isso, exclui-se da análise apólices de pessoas jurídicas e/ou coletivas). Este corte se justifica pela teoria econômica subjacente, que aponta os custos de procura individuais como fonte da fricção causadora de dispersão de preços neste mercado.

Apenas contratos com cobertura compreensiva fazem parte da amostra analisada. Tais contratos correspondem a aproximadamente $97 \%$ dos contratos vendidos neste mercado. Este corte exclui da análise contratos que cobrem apenas incêndio e roubo, mas que não cobrem colisão. Por fim, a amostra inclui apenas veículos populares com até 3 anos de uso. Chamamos de "populares" aqueles veículos que possuem motor 1.0, cujos modelos na amostra são: Gol, Celta, Corsa, 
Uno, Palio, Fiesta, Ka e Clio. Os contratos de seguros de veículos populares representam quase $50 \%$ do mercado. Ao todo, a amostra analisada neste trabalho contém 2,2 milhões de observações.

Uma vez definida a amostra, surge uma nova questão relevante: quais variáveis devem ser incluídas na análise? Devemos incluir na análise todas as variáveis relevantes e observáveis pelo corretor no instante em que escolhe sua taxa de comissão. A Tabela 1 apresenta todas as variáveis que possam afetar a decisão do corretor e que se encontram disponíveis na base de dados da SUSEP.

Tabela 1. Descrição das Variáveis

\begin{tabular}{|c|c|}
\hline Variável & Descrição \\
\hline Taxa de Comissão & Taxa de comissão escolhida pelo corretor ao vender o contrato. \\
\hline Prêmio da Seguradora & $\begin{array}{l}\text { Prêmio recebido pela seguradora. Igual ao prêmio total pago pelo segurado menos } \\
\text { a remuneração do corretor. }\end{array}$ \\
\hline Tipo de Franquia & $\begin{array}{l}\text { Dummy que assume valor } 1 \text { para contratos com franquia reduzida, e } 0 \text { para contra- } \\
\text { tos com franquia normal. }\end{array}$ \\
\hline Importância Segurada & $\begin{array}{l}\text { Valor da importância segurada do veículo. A importância segurada representa } 0 \\
\text { limite máximo de indenização em casco. }\end{array}$ \\
\hline Sexo & $\begin{array}{l}\text { Dummy que assume valor } 1 \text { para segurados do sexo masculino, e } 0 \text { para segurados } \\
\text { do sexo feminino. }\end{array}$ \\
\hline Idade & Idade do motorista medida em anos. \\
\hline Classe de Bônus & $\begin{array}{l}\text { Dummies que indicam a classe de bônus do segurado. Quanto maior a classe de } \\
\text { bônus, maior o desconto concedido pela seguradora sobre o prêmio do seguro. }\end{array}$ \\
\hline Modelo do Veículo & $\begin{array}{l}\text { Dummies que indicam o modelo do veículo. Sendo eles: Gol, Celta, Corsa, Uno, } \\
\text { Palio, Fiesta, Ka, e Clio. Todos com motor 1.0. }\end{array}$ \\
\hline Ano/Modelo & Dummies que indicam o ano do modelo do veículo. \\
\hline Semestre & Dummies que indicam o semestre no qual o contrato foi transacionado. \\
\hline CEP & Dummies para o Código de Endereçamento Postal (CEP) do segurado. \\
\hline
\end{tabular}

A Tabela 2 apresenta algumas estatísticas descritivas para as variáveis quantitativas. As estatísticas descritivas escolhidas são: média, desvio-padrão, além dos percentis 25\%,50\% (mediana) e 75\%. 
Tabela 2. Estatísticas Descritivas

\begin{tabular}{lccccc}
\hline \multirow{2}{*}{ Variável } & Média & DP & \multicolumn{3}{c}{ Percentis } \\
\cline { 4 - 6 } & & & $25 \%$ & $50 \%$ & $75 \%$ \\
\hline Taxa de Comissão & 0,1695 & 0,1042 & 0,10 & 0,195 & 0,25 \\
Prêmio da Seguradora & 590,85 & 265,99 & 420,75 & 549,22 & 720,75 \\
Importância Segurada & $16.608,81$ & $3.810,95$ & 13.886 & 15.995 & 18.678 \\
Percentual de Bônus & 13,43 & 13,35 & 0 & 10 & 25 \\
Valor da Franquia & 725,71 & 333,54 & 450 & 800 & 900 \\
Idade & 42,31 & 12,52 & 33 & 41 & 51 \\
\hline
\end{tabular}

A variável "taxa de comissão" equivale exatamente à variável $\alpha$ no modelo teórico da Seção 3. Ou seja, trata-se da taxa de comissão escolhida pelo corretor no ato da venda do contrato. A taxa média de comissão na amostra é igual a 16,95\%, com desvio-padrão igual a $10,42 \%$. A Figura 1 apresenta o histograma das taxas de comissão.

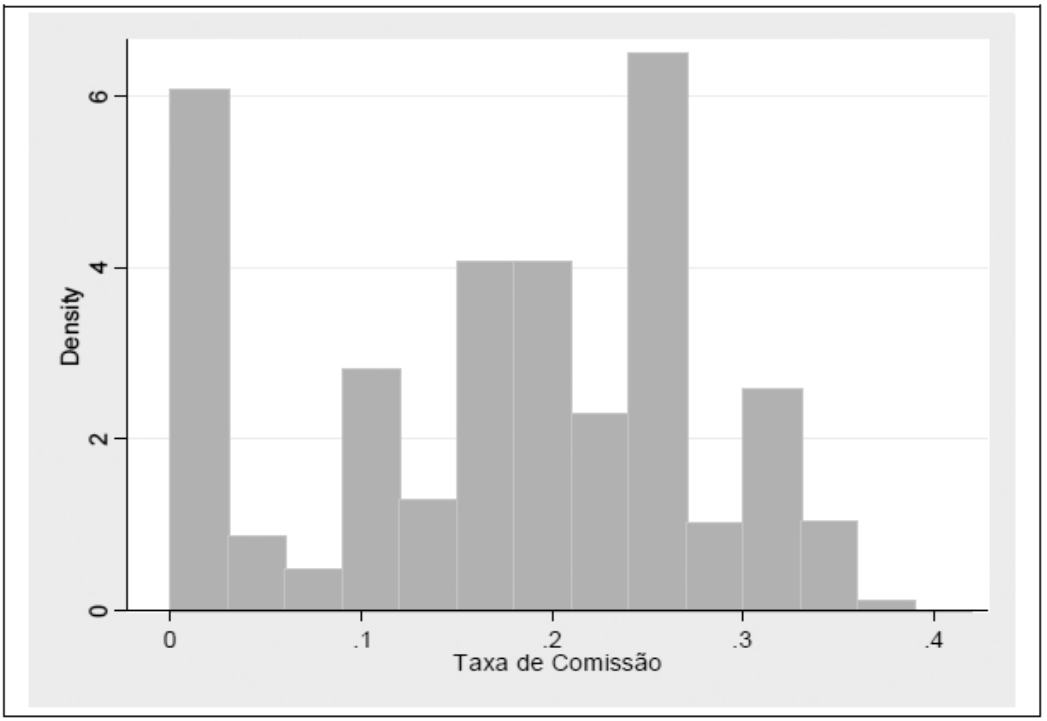

A variável "prêmio da seguradora" equivale exatamente à variável P no modelo teórico da Seção 3. Ou seja, é igual ao prêmio total pago pelo segurado menos a remuneração do corretor. O prêmio médio recebido pelas seguradoras na amostra foi $\mathrm{R} \$ 590,85$, com desvio-padrão igual a $R \$ 265,99$. A Figura 2 apresenta o histograma do prêmio da seguradora. 


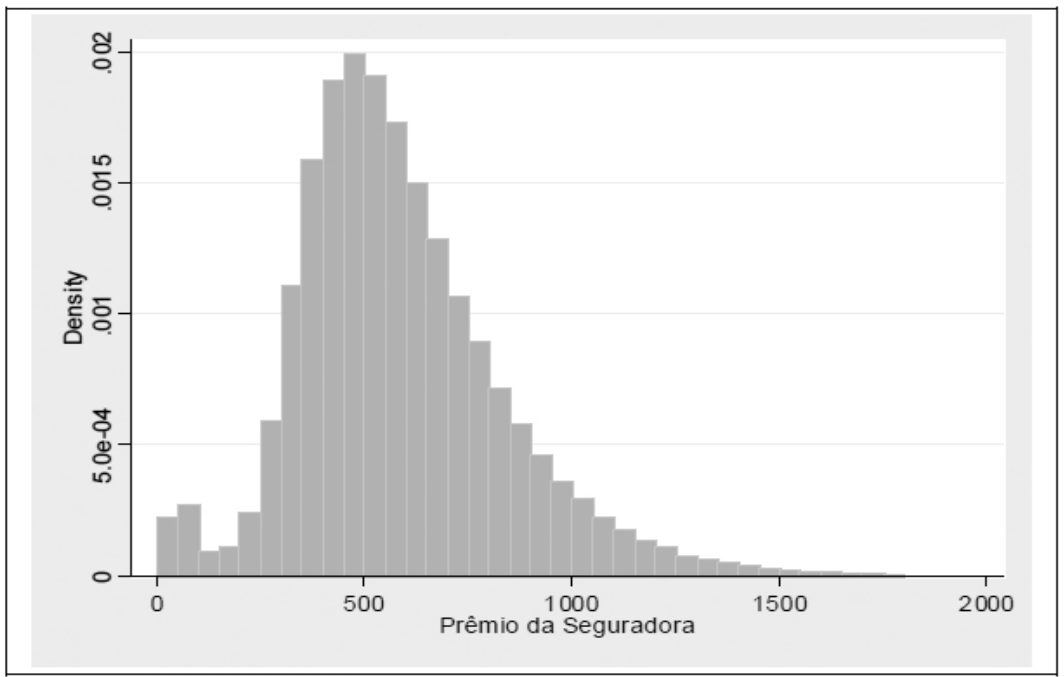

A variável "tipo de franquia" representa a franquia contratada. Temse que aproximadamente $60 \%$ dos segurados na amostra optaram por contratar franquia normal, enquanto os demais optaram por franquias reduzidas. O valor médio das franquias na amostra foi $\mathrm{R} \$ 725,71$, com desvio-padrão de $\mathrm{R} \$ 333,54$.

A variável "importância segurada" indica o limite máximo de indenização para o veículo. Em geral, este valor está muito próximo do valor de mercado do veículo. $\mathrm{Na}$ amostra analisada a importância segurada média foi de $\mathrm{R} \$ 16.608,81$, com desvio-padrão de $\mathrm{R} \$ 3.810,95$.

A inclusão das variáveis "tipo de franquia" e "importância segurada" na análise têm como objetivo controlar pelas características do contrato.

A variável "sexo" indica o sexo do segurado. Tem-se que 54\% dos contratos na amostra são de segurados do sexo masculino.

A variável "idade" representa a idade do segurado, medida em anos. A idade média na amostra foi de 42,31 anos, com desvio-padrão de 12,52 anos.

Construiu-se uma dummy para cada classe de bônus. As seguradoras utilizam a seguinte regra para as classes de bônus: o segurado sobe uma classe de bônus a cada ano que passar sem declarar sinistro. 
Quanto maior a classe de bônus, maior o desconto que a seguradora concede sobre o prêmio do seguro. O bônus é pessoal e intransferível entre segurados, no entanto o segurado pode carregar seu bônus de uma seguradora para outra. Na amostra, a taxa média de desconto decorrente das classes de bônus foi de 13,43\%, com desvio-padrão de $13,35 \%$. A inclusão das variáveis "sexo", "idade" e "classe de bônus" têm como objetivo controlar pelas características do segurado. Construíram-se dummies para os diferentes modelos de veículos populares. Os veículos mais frequentes na amostra são Gol (23\%), Palio (23\%) e Celta (17\%). Também se construíram dummies para o ano/ modelo dos veículos. O objetivo desse grupo de variáveis é controlar pelas características do veículo.

Criaram-se também dummies por semestre em que o contrato foi transacionado. O objetivo dessas variáveis é controlar o fator tempo na decisão do corretor. Criaram-se também dummies por código de endereçamento postal (CEP) do segurado. Esta variável permite controlar todos os aspectos relacionados ao local de residência do segurado, tais como o risco exógeno de roubo e a renda média do bairro.

\section{Nota sobre a Ausência de Algumas Variáveis}

Cada seguradora no mercado desenvolve um questionário para inferir sobre o perfil de risco de seus segurados. Algumas questões comumente presentes nesses questionários são relativas à: estado civil, número de filhos, distância entre a residência e o local de trabalho, garagem etc. No entanto, estes questionários não são padronizados entre as seguradoras e a SUSEP não coleta tais dados.

Pode-se argumentar que a ausência desses dados, que são observados pelos corretores, pode enviesar os resultados dos testes. Porém, este argumento contém uma falha. Isto porque o prêmio determinado pela seguradora já contém toda informação relevante sobre os questionários de risco. Nas palavras de Cohen (2005):

"I will for simplicity use the premium of the policyholders instead of their characteristics. In all regressions in this paper, I have verified that the results are robust and hold either using the characteristics of the policyholder or using the premium instead." 
Sendo assim, a inclusão do prêmio da seguradora nas regressões deverá controlar por todas as características dos segurados contidas nos questionários.

\subsection{Testando se a Taxa de Comissão decresce com o Prêmio da Seguradora}

O Lema 4 prevê que o valor esperado das taxas de comissão deve decrescer com o prêmio fixado pela seguradora. Para testar esta previsão regredimos a taxa de comissão (em nível e logaritmo) contra o prêmio fixado pela seguradora (em logaritmo). Utilizando a amostra descrita na Seção 4, estimaram-se por mínimos quadrados ordinários (MQO) as seguintes funções de regressão populacional:

$$
\begin{aligned}
& E[\alpha \mid \ln P, \mathrm{X}]=\beta_{0}+\beta_{1} \ln P+\beta_{2} \mathrm{X} \\
& E[\ln \alpha \mid \ln P, \mathrm{X}]=\delta_{0}+\delta_{1} \ln P+\delta_{2} \mathrm{X}
\end{aligned}
$$

Portanto, os coeficientes de interesse são $\beta_{1}$ e $\delta_{1}$. Evidências de que tais coeficientes sejam negativos estariam corroborando a previsão do modelo. A variável $\alpha$, tal como no modelo teórico, representa a taxa de comissão escolhida pelo corretor. A variável lnP representa o logaritmo do prêmio da seguradora, e o vetor X representa um conjunto de variáveis observáveis pelo corretor no instante em que escolheu sua taxa de comissão. A descrição completa das variáveis utilizadas nos testes se encontra na Tabela 1.

Nota técnica: pode-se observar na Figura 1 que as taxas de comissão não apresentam distribuição aproximadamente normal. No entanto, dado que amostra é bastante grande assumiremos válidas as propriedades assimptóticas de MQO. A metodologia de teste resumida pelas Equações (12) e (13) foram aplicadas, separadamente, em todas as 41 classes de localização que dividem o mercado nacional.

A Tabela 3 apresenta os coeficientes estimados, $\beta_{1}$ e $\delta_{1}$, para cada classe de localização. Para simplificar a apresentação, optou-se por omitir os coeficientes estimados das variáveis de controle. 
Tabela 3 - Regressões da Taxa de Comissão em Nível e Logaritmo, por Classe de Localização

\begin{tabular}{|c|c|c|c|}
\hline & $\begin{array}{l}\mathrm{E}(\alpha \mid \ln \mathrm{P}, \mathrm{X})=\beta_{0}+\beta_{1} \ln \mathrm{P}+\beta_{2} \mathrm{X} \\
\mathrm{E}(\ln \alpha \mid \ln \mathrm{P}, \mathrm{X})=\delta_{0}+\delta_{1} \ln \mathrm{P}+\delta_{2} \mathrm{X}\end{array}$ & & \\
\hline Classe & Localização & & \\
\hline 1 & Região metropolitana de Porto Alegre e metropolitana de Caxias do Sul & $\begin{array}{l}-0.0853^{\star \star \star} \\
(0,0013)\end{array}$ & $\begin{array}{l}-0.3688^{\star \star \star} \\
(0,0091)\end{array}$ \\
\hline 2 & Demais regióes do Rio Grande do Sul & $\begin{array}{l}-0.0762^{\star * \star} \\
(0,0018)\end{array}$ & $\begin{array}{l}-0.5194^{\star \star \star} \\
(0,0147)\end{array}$ \\
\hline 3 & Região metropolitana de Florianópolis e sul de Santa Catarina & $\begin{array}{l}-0.0704^{* \star \star} \\
(0,0015)\end{array}$ & $\begin{array}{l}-0.7282^{\star \star \star} \\
(0,0148)\end{array}$ \\
\hline 4 & Oeste catarinense & $\begin{array}{l}-0.0915^{* \star \star} \\
(0,0032)\end{array}$ & $\begin{array}{l}-0.7664^{\star \star \star} \\
(0,0304)\end{array}$ \\
\hline 5 & Blumenau e demais regiões de Santa Catarina & $\begin{array}{l}-0.0595^{\star \star \star} \\
(0,0019)\end{array}$ & $\begin{array}{l}-0.4608^{\star \star \star} \\
(0,0148)\end{array}$ \\
\hline 6 & Foz do Iguaçu, Medianeira, Cascavél e Toledo & $\begin{array}{l}-0.0726^{\star \star \star} \\
(0,0032)\end{array}$ & $\begin{array}{l}-0.5285^{\star \star \star} \\
(0,0295)\end{array}$ \\
\hline 7 & Região metropolitana de Curitiba & $\begin{array}{l}-0.0360^{\star * \star} \\
(0,0009)\end{array}$ & $\begin{array}{l}-0.2330^{\star \star \star} \\
(0,0070)\end{array}$ \\
\hline 8 & Demais regiões do Paraná & $\begin{array}{l}-0.0253^{* * \star} \\
(0,0013)\end{array}$ & $\begin{array}{l}-0.2840^{\star \star *} \\
(0,0114)\end{array}$ \\
\hline 9 & Vale do Paraíba e Vale do Ribeira & $\begin{array}{l}-0.0385^{\star \star \star} \\
(0,0007)\end{array}$ & $\begin{array}{l}-0.1773^{\star \star \star} \\
(0,0055)\end{array}$ \\
\hline 10 & Baixada Santista e litoral norte de São Paulo & $\begin{array}{l}-0.0398^{\star \star \star} \\
(0,0008)\end{array}$ & $\begin{array}{l}-0.1641^{\star \star \star} \\
(0,0056)\end{array}$ \\
\hline 11 & Região metropolitana de São Paulo & $\begin{array}{l}-0.0237^{\star \star \star} \\
(0,0002)\end{array}$ & $\begin{array}{l}-0.0953^{\star \star \star} \\
(0,0016)\end{array}$ \\
\hline 12 & Campinas e região & $\begin{array}{l}-0.0240^{\star * \star} \\
(0,0005)\end{array}$ & $\begin{array}{l}-0.1518^{\star \star \star} \\
(0,0034)\end{array}$ \\
\hline 13 & Demais regiões do Estado de São Paulo & $\begin{array}{l}-0.0297^{\star \star \star \star} \\
(0,0003)\end{array}$ & $\begin{array}{l}-0.1711^{\star \star \star} \\
(0,0023)\end{array}$ \\
\hline 14 & Triângulo Mineiro e Alto Paranaíba & $\begin{array}{l}-0.0254^{\star \star \star} \\
(0,0020)\end{array}$ & $\begin{array}{l}-0.3854^{\star \star \star} \\
(0,0181)\end{array}$ \\
\hline 15 & Sul, Sudoeste, Oeste e Central de Minas Gerais & $\begin{array}{l}-0.0482^{\star \star \star} \\
(0,0022)\end{array}$ & $\begin{array}{l}-0.5200^{\star \star \star} \\
(0,0212)\end{array}$ \\
\hline 16 & $\begin{array}{l}\text { Regiao metropolitana de Belo Horizonte, Zona da Mata, Campo das Vertentes e Norte e } \\
\text { Noroeste de MG }\end{array}$ & $\begin{array}{l}-0.0277^{* * \star} \\
(0,0009)\end{array}$ & $\begin{array}{l}-0.2742^{\star \star \star} \\
(0,0075)\end{array}$ \\
\hline 17 & Vale do Rio Doce, Vale do Mucuri e Vale do Jequitinhonha & $\begin{array}{l}-0.0432^{* \star \star} \\
(0,0030)\end{array}$ & $\begin{array}{l}-0.3304^{\star \star \star} \\
(0,0256)\end{array}$ \\
\hline 18 & Região metropolitana do Rio de Janeiro & $\begin{array}{l}-0.0482^{\star \star \star} \\
(0,0007)\end{array}$ & $\begin{array}{l}-0.2951^{\star \star \star} \\
(0,0057)\end{array}$ \\
\hline 19 & Interior do Rio de Janeiro & $\begin{array}{l}-0.0430^{* * *} \\
(0,0017)\end{array}$ & $\begin{array}{l}-0.3398^{\star \star *} \\
(0,0167)\end{array}$ \\
\hline 20 & Estado do Espírito Santo & $\begin{array}{l}-0.0412^{\star \star \star} \\
(0,0020)\end{array}$ & $\begin{array}{l}-0.2480^{\star \star \star} \\
(0,0182)\end{array}$ \\
\hline
\end{tabular}


(Tabela 3. Continuação)

\begin{tabular}{|c|c|c|c|c|}
\hline Classe & & Localização & & \\
\hline 21 & Estado da Bahia & & $\begin{array}{l}-0.0487^{\star \star \star} \\
(0,0012)\end{array}$ & $\begin{array}{l}-0.5519^{* \star *} \\
(0,0124)\end{array}$ \\
\hline 22 & Estado do Sergipe & & $\begin{array}{l}-0.0202^{\star \star \star} \\
(0,0029)\end{array}$ & $\begin{array}{l}-0.4501^{\star \star *} \\
(0,0351)\end{array}$ \\
\hline 23 & Estado de Pernambuco & & $\begin{array}{l}-0.0370^{\star \star \star} \\
(0,0014)\end{array}$ & $\begin{array}{l}-0.5262^{\star \star \star} \\
(0,0139)\end{array}$ \\
\hline 24 & Estado da Paraíba & & $\begin{array}{l}-0.0560^{\star \star \star} \\
(0,0027)\end{array}$ & $\begin{array}{l}-0.8646^{* \star *} \\
(0,0324)\end{array}$ \\
\hline 25 & Estado do Rio Grande do Norte & & $\begin{array}{l}-0.0284^{\star \star \star} \\
(0,0023)\end{array}$ & $\begin{array}{l}-0.3920^{\star \star \star} \\
(0,0230)\end{array}$ \\
\hline 26 & Estado de Alagoas & & $\begin{array}{l}-0.0068^{*} \\
(0,0029)\end{array}$ & $\begin{array}{l}-0.4007^{\star \star \star} \\
(0,0273)\end{array}$ \\
\hline 27 & Estado do Ceará & & $\begin{array}{l}-0.0567^{\star \star \star} \\
(0,0019)\end{array}$ & $\begin{array}{l}-0.5960^{\star \star *} \\
(0,0190)\end{array}$ \\
\hline 28 & Estado do Piauí & & $\begin{array}{l}-0.0403^{\star \star \star} \\
(0,0044)\end{array}$ & $\begin{array}{l}-0.4861^{\star \star \star} \\
(0,0504)\end{array}$ \\
\hline 29 & Estado do Maranhão & & $\begin{array}{l}-0.0699^{\star \star \star} \\
(0,0032)\end{array}$ & $\begin{array}{l}-0.5739 * \star * \\
(0,0390)\end{array}$ \\
\hline 30 & Estado do Pará & & $\begin{array}{l}-0.0667^{\star \star \star} \\
(0,0028)\end{array}$ & $\begin{array}{l}-0.4028^{\star \star \star} \\
(0,0251)\end{array}$ \\
\hline 31 & Estado do Amazonas & & $\begin{array}{l}-0.0481^{\star \star \star} \\
(0,0039)\end{array}$ & $\begin{array}{l}-0.4387^{* * *} \\
(0,0349)\end{array}$ \\
\hline 32 & Estado do Amapá & & $\begin{array}{l}-0.0429^{\star \star \star} \\
(0,0050)\end{array}$ & $\begin{array}{l}-0.2696^{\star \star \star} \\
(0,0465)\end{array}$ \\
\hline 33 & Estado de Rondônia & & $\begin{array}{l}-0.0413^{\star \star \star} \\
(0,0051)\end{array}$ & $\begin{array}{l}-0.4148^{\star \star *} \\
(0,0600)\end{array}$ \\
\hline 34 & Estado de Roraima & & $\begin{array}{l}-0,0208 \\
(0,0107)\end{array}$ & $\begin{array}{l}-0.8421^{\star \star \star} \\
(0,1094)\end{array}$ \\
\hline 35 & Estado do Acre & & $\begin{array}{l}-0.0224^{\star} \\
(0,0096)\end{array}$ & $\begin{array}{l}-0.4810^{\star \star *} \\
(0,1245)\end{array}$ \\
\hline 36 & Estado do Mato Grosso & & $\begin{array}{l}-0.0092^{\star \star \star} \\
(0,0025)\end{array}$ & $\begin{array}{l}-0.2148^{\star \star \star} \\
(0,0228)\end{array}$ \\
\hline 37 & Estado do Mato Grosso do Sul & & $\begin{array}{l}-0.0130^{\star \star \star} \\
(0,0026)\end{array}$ & $\begin{array}{l}-0.2931^{\star \star \star} \\
(0,0279)\end{array}$ \\
\hline 38 & Distrito Federal & & $\begin{array}{l}-0.0207^{\star \star \star} \\
(0,0013)\end{array}$ & $\begin{array}{l}-0.2635^{\star \star \star} \\
(0,0118)\end{array}$ \\
\hline 39 & Estado de Goiás & & $\begin{array}{l}-0.0560^{\star \star \star} \\
(0,0021)\end{array}$ & $\begin{array}{l}-0.4348^{* \star *} \\
(0,0212)\end{array}$ \\
\hline 40 & Estado de Tocantins & & $\begin{array}{l}-0.0673^{\star \star \star} \\
(0,0052)\end{array}$ & $\begin{array}{l}-0.9640^{* \star *} \\
(0,0930)\end{array}$ \\
\hline 41 & Sudeste de Goiás & & $\begin{array}{l}-0,0052 \\
(0,0036)\end{array}$ & $\begin{array}{l}-0.3441^{* * *} \\
(0,0441)\end{array}$ \\
\hline
\end{tabular}

*** Significante a $0,1 \%$. Erros-padrão robustos entre parênteses. 
Nas regressões da taxa de comissão em nível (coluna $\beta_{1}$ ) todos os 41 coeficientes estimados apresentaram sinal negativo, dos quais 37 apresentaram significância estatística a $0,1 \%$ e outros 2 coeficientes apresentaram significância a 5\%.

Nas regressões da taxa de comissão em logaritmo (coluna $\delta_{1}$ ), todos os 41 coeficientes estimados apresentaram sinal negativo e significância estatística a $0,1 \%$. Conclui-se então que os resultados apresentados na Tabela 3 corroboram de forma bastante robusta o Lema 4 .

\section{Análise dos Resultados para a Região Metropolitana de São Paulo}

Apresentar e analisar os resultados das 41 classes de localização separadamente seria uma tarefa bastante repetitiva. Então, optou-se por apresentar e analisar detalhadamente apenas os resultados obtidos no principal mercado brasileiro, a região metropolitana de São Paulo (classe de localização 11), que corresponde sozinha por aproximadamente 22\% dos contratos de seguros de automóveis vendidos no Brasil.

As estimativas das Equações (12) e (13) para a região metropolitana de São Paulo são apresentadas na Tabela 4. 
Tabela 4. Regressões da Taxa de Comissão em Nível e Logaritmo Apenas Região Metropolitana de São Paulo

\begin{tabular}{|c|c|c|}
\hline Var. Dependente: Taxa de Comissão & Em nível & Em logaritmo \\
\hline \multirow{2}{*}{ Logaritmo do Prêmio (InP) } & $-0,0237^{\star \star \star *}$ & $-0,0953^{* * *}$ \\
\hline & $(0,0002)$ & $(0,0016)$ \\
\hline \multirow{2}{*}{ Tipo de Franquia } & $-0,0111^{* \star *}$ & $-0,0277^{\star \star \star}$ \\
\hline & $(0,0005)$ & $(0,0043)$ \\
\hline \multirow{2}{*}{ Idade } & $3,16 \mathrm{E}-05$ & 0,0001 \\
\hline & $(4,01 \mathrm{E}-07)$ & $(2,35 \mathrm{E}-06)$ \\
\hline \multirow{2}{*}{ Sexo } & $-0,0035^{\star \star *}$ & $-0,0004$ \\
\hline & $(0,0003)$ & $(0,0021)$ \\
\hline \multirow{2}{*}{ Importância Segurada } & $0,0100 * \star \star$ & $0,0556^{\star \star \star}$ \\
\hline & $(0,0010)$ & $(0,0072)$ \\
\hline \multirow{2}{*}{ Classe de Bônus 1} & $0,0338^{\star * *}$ & $-0,006$ \\
\hline & $(0,0004)$ & $(0,0035)$ \\
\hline \multirow{2}{*}{ Classe de Bônus 2} & $0,0445^{\star \star \star}$ & $0,0715^{\star \star \star}$ \\
\hline & $(0,0005)$ & $(0,0040)$ \\
\hline \multirow{2}{*}{ Classe de Bônus 3} & $0,0379 * \star *$ & $0,0584^{\star \star \star}$ \\
\hline & $(0,0006)$ & $(0,0043)$ \\
\hline \multirow{2}{*}{ Classe de Bônus 4} & $0,0424^{\star \star *}$ & 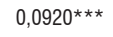 \\
\hline & $(0,0006)$ & $(0,0043)$ \\
\hline \multirow{2}{*}{ Classe de Bônus 5} & $0,0336^{\star \star *}$ & $0,0772^{\star \star \star}$ \\
\hline & $(0,0005)$ & $(0,0034)$ \\
\hline \multirow{2}{*}{ Classe de Bônus 6} & $0,0384^{* * *}$ & $0,0320 * * *$ \\
\hline & $(0,0006)$ & $(0,0049)$ \\
\hline \multirow{2}{*}{ Classe de Bônus 7} & $0,0326^{\star * *}$ & 0,0002 \\
\hline & $(0,0007)$ & $(0,0048)$ \\
\hline \multirow{2}{*}{ Classe de Bônus 8} & $0,0914^{\star \star \star}$ & $0,2622^{\star \star \star}$ \\
\hline & $(0,0012)$ & $(0,0075)$ \\
\hline \multirow{2}{*}{ Ano/Modelo 2002} & $0,0029 * \star \star$ & $0,0241^{\star \star \star}$ \\
\hline & $(0,0004)$ & $(0,0026)$ \\
\hline \multirow{2}{*}{ Ano/Modelo 2003} & $0,0143^{\star \star *}$ & $0,0549 * \star \star$ \\
\hline & $(0,0005)$ & $(0,0036)$ \\
\hline \multirow{2}{*}{ Ano/Modelo 2004} & $0,0259 * \star \star$ & $0,0690 * \star \star$ \\
\hline & $(0,0009)$ & $(0,0067)$ \\
\hline Modelo do Veículo & 8 dummies & 8 dummies \\
\hline Semestre & 4 dummies & 4 dummies \\
\hline CEP & 6656 & 6501 \\
\hline Tamanho da Amostra & 464.320 & 394.990 \\
\hline R2 Ajustado & $11,04 \%$ & $3,62 \%$ \\
\hline
\end{tabular}

*** Significante a 0,1\%. Erros-padrão robustos entre parênteses.

Regressão inclui termo constante. 
A regressão da taxa de comissão em nível indica que a cada $1 \%$ de aumento no prêmio mínimo fixado pela seguradora a taxa de comissão do corretor diminui 0,0237 pontos percentuais em média. Vale lembrar que o prêmio final a ser pago pelo segurado é dado por $(1+\alpha) \mathrm{P}$, portanto caso a seguradora aumente o prêmio mínimo em $1 \%$ então o prêmio final pago pelo segurado aumentará, em média, menos do que $1 \%$ (o aumento do prêmio final seria igual a $1 \%$ somente se a taxa de comissão permanecesse constante).

Pode-se argumentar que a taxa de comissão não seja função linear de variações percentuais no prêmio mínimo. Então, a regressão da taxa de comissão em logaritmo nos permite obter a elasticidade da taxa de comissão do corretor em relação ao prêmio mínimo fixado pela seguradora. O coeficiente estimado sugere que a cada $1 \%$ de aumento no prêmio mínimo a taxa de comissão diminui 0,0953\%, em média. Vale lembrar que a receita total do corretor é $\alpha \mathrm{P}$ e, portanto, deve aumentar em função de aumentos no prêmio mínimo.

Dentre as demais variáveis de controle, vale mencionar que o valor esperado das taxas de comissão é maior nos contratos com classes de bônus diferentes de zero (a base de comparação são os contratos com classe zero de bônus). Uma possível explicação para este resultado é que segurados com maiores classes de bônus já estão no mercado há bastante tempo e, possivelmente, já foram fidelizados por algum corretor que esteja lhe extraindo o excedente.

Os $\mathrm{R}^{2}$ ajustados indicam que as variáveis observáveis pelo corretor explicam apenas $11,04 \%$ e 3,62\% da variação nas taxas de comissão em nível e logaritmo, respectivamente. Os baixos valores para os $\mathrm{R}^{2}$ sugerem que a maior parte da dispersão nas taxas de comissão seja explicada por fatores não observáveis. Este resultado também fortalece o modelo da Seção 3, pois indica que os fatores não observáveis, como o custo de procura dos segurados, influenciam bastante a escolha do corretor.

Por fim, vale observar que o tamanho da amostra é menor na regressão da taxa de comissão em logaritmo. Isto se deve ao fato de alguns corretores escolherem taxa zero de comissão, e nestes casos o logaritmo é indefinido. 


\section{Conclusão}

Este artigo apresentou uma versão do modelo de Stahl (1989) para explicar como a taxa de comissão escolhida pelos corretores de seguros depende do prêmio requerido pela seguradora, do custo de procura dos segurados e do número de corretores competindo no mercado.

O modelo prevê que, ceteris paribus, o valor esperado das taxas de comissão decresce com o prêmio requerido pela seguradora. Os resultados empíricos apresentados neste artigo sugerem que esta predição se verifica no mercado brasileiro.

Na prática, este artigo contribui para que as seguradoras tenham maior conhecimento sobre o comportamento estratégico dos corretores. Desta forma, as seguradoras terão maior controle sobre o prêmio final e, consequentemente, sobre a alocação do risco nos contratos de seguro.

Este artigo, por ser um dos primeiros a estudar a relação corretor/ segurado e corretor/seguradora, abre um grande leque de oportunidades de pesquisas futuras. Por exemplo, os resultados da regressão expressos na Tabela 4 sugerem que os corretores cobram maiores taxas de comissão dos segurados com maiores classes de bônus. Este resultado pode ser um indício de que os corretores, de alguma forma, estejam fidelizando os segurados nos primeiros anos para, em seguida, extrair-lhes mais excedente. Este tipo de análise só será possível através de modelos dinâmicos com custos de troca (switching costs).

\section{Referências}

Arnott, R. e Stiglitz, J. (1988). The Basic Analytics of Moral Hazard. Scandinavian Journal of Economics, 90(3), 383-413.

Aurichio, B. C. e Braido, L. H. B. (2010). Dynamic Price Competition in Auto-Insurance Brokerage. Tese de Doutorado, Escola de Pós-Graduação em Economia (EPGE-FGV), Capítulo 1, 2-33.

Barron, J. M., Taylor, B. A. e Umbeck, J. R. (2004). Number of sellers, average prices, and price dispersion. International Journal of Industrial Organization, 22(8-9), 1041-1066.

Benabou, R., e Gertner, R. (1993). Search with Learning from Prices: Does Increased Inflationary Uncertainty Lead to Higher Markups. The Review of Economic Studies, 60(1), 69-93.

Burdett, K., e Judd, K. L. (1983). Equilibrium Price Dispersion. Econometrica, 51(4), 955-970. 
Carlson, J. A., e McAfee, R. P. (1983). Discrete Equilibrium Price Dispersion. The Journal of Political Economy, 91(3), 480-493.

Chandra, A. e Tappata, M. (2009). Consumer Search and Dynamic Price Dispersion. An Application to Gasoline Markets. Working paper.

Chiappori, P. A., Julien, B., Salanié, B. e Salanié, F. (2006). Asymmetric Information in Insurance: General Testable Implications. RAND Journal of Economics, 37(4), 783-798.

Dahlby, B., e West, D. S. (1986). Price Dispersion in an Automobile Insurance Market. The Journal of Political Economy, 94(2), 418-438.

Diamond, P. A. (1971). A model of price adjustment. Journal of Economic Theory, 3(2), 156-168.

MacMinn, R. D. (1980). Search and Market Equilibrium. The Journal of Political Economy, 88(2), 308-327.

Reinganum, J. F. (1979). A Simple Model of Equilibrium Price Dispersion. The Journal of Political Economy, 87(4), 851-858.

Rothschild, M. e Stiglitz, J. (1976). Equilibrium in Competitive Insurance Markets: An Essay on the Economics of Imperfect Information. Quarterly Journal of Economics, 90(4), 629-49.

Sorensen, A. T. (2000). Equilibrium Price Dispersion in Retail Markets for Prescription Drugs. Journal of Political Economy, 108(4), 833-850.

Stahl, Dale O. (1989). Olipolistic pricing with sequential consumer search. American Economic Review, 79(4), 700-712.

Stigler, G. (1961). The economics of information. Journal of Political Economy, 69, 213-225. 